\title{
Phase field assisted analysis of a solidification based metal refinement process
}

\author{
A. Viardin*, B. Böttger and M. Apel
}

*Correspondence:

a.viardin@access-technology.de ACCESS e.V., Intzestrasse 5, D-52072

Aachen, Germany

\begin{abstract}
Ultra pure metals have various applications, e. g. as electrical conductors. Crystallization from the melt, e. g. via zone melting, using the segregation of impurities at the solidification front is the basic mechanism behind different technical processes for the refining of metals and semi-metals. In this paper, we focus on a crystallization methodology with a gas cooled tube ("cooled finger") dipped into a metallic melt in a rotating crucible. The necessary requirement for purification in a solidification process is a morphologically stable solidification front. This is the only way to enable macroscopic separation of the impurities, e. g. by convection. For cellular or dendritic solidification morphologies, the segregated impurities are trapped into the interdendritic melt and remain as microsegregations in the solidified metal. Morphological stability depends on the temperature gradient $\mathrm{G}$ at the solidification front, the solidification front velocity $V_{\text {front }}$ and thermodynamic alloy properties like the segregation coefficients of the impurity elements. To quantify the impact of these parameters on the morphological evolution, especially on the planar/cellular transition and thus on microsegregation profiles, phase field simulations coupled to a thermodynamic database are performed for an aluminium melt with three impurities, $\mathrm{Si}, \mathrm{Mn}$ and Fe. In particular, we have investigated the morphology evolution from the start of solidification at the cooled finger towards a stationary growth regime, because in the technical process a significant fraction of the melt solidifies along the initial transient. To solve the transient long range temperature evolution on an experimental length scale, the temperature field has been calculated using the homoenthalpic approach together with a 1D temperature field approximation. The simulations provide the process window for an energy efficient purification process, i. e. low thermal gradients, and elucidate the benefit of melt convection.
\end{abstract}

Keywords: Alloy solidification, Dendritic growth, Aluminum, Planar growth, Phase field method, Purification, Cooled finger

\section{Introduction}

Pure and ultra pure aluminum has various applications in the electronic industry (Curtolo et al. 2021). Pure aluminum is used as foils for the production of electrolytic capacitors. Ultra pure aluminum is used for computer storage hard disk or used as a sputtered coating of integrated circuits.

(c) The Author(s). 2022 Open Access This article is licensed under a Creative Commons Attribution 4.0 International License, which permits use, sharing, adaptation, distribution and reproduction in any medium or format, as long as you give appropriate credit to the original author(s) and the source, provide a link to the Creative Commons licence, and indicate if changes were made. The images or other third party material in this article are included in the article's Creative Commons licence, unless indicated otherwise in a credit line to the material. If material is not included in the article's Creative Commons licence and your intended use is not permitted by statutory regulation or exceeds the permitted use, you will need to obtain permission directly from the copyright holder. To view a copy of this licence, visit http://creativecommons.org/licenses/by/4.0/. 
Hence, an energy efficient method (efficient by low energy consumption) for further purification of metallic aluminum is of technical interest. Zone melting and its different technological variants are commonly used methods to produce ultra pure semiconductors or metals (Zhang et al. 2018). Its limit is defined by the thermodynamic properties of the alloy system, in particular by the segregation coefficients of the different elements. In this paper, we will investigate the potential of a so called "cooled finger" solidification device (see Fig. 1, the explanation of its operation is given further in "Cooled finger" section) for the purification of aluminum with multiple impurities via phase-field simulations. A necessary requirement and thus central for an efficient purification process is to maintain a planar, morphologically stable solid/liquid interface in a multicomponent system (Guillemot and Gandin 2021, Lahari and Choudhury2017). Only in this case, the theoretical limit of a solidification based purification method as given by the segregation coefficient can be achieved. Therefore, the physical phenomenon to be investigated is the morphological stability of a moving solid/liquid interface. A second requirement is an efficient, long range transport of the impurity in the melt, e. g. by convection. Hence, the interrelation between the diffusive boundary layer in the liquid close to the solidification front and the morphological stability will be investigated as well. The solidification process can be divided into two stages. The first stage is characterized by a dynamic temperature evolution while cooling down of the melt triggers the nucleation of fcc-Al on the cooled finger. The second stage can be fairly well approximated by a rather stationary temperature field, i. e. by a constant temperature gradient and cooling rate resulting in a constant velocity of the liquidus isotherm. Both stages are investigated using individual simulation scenarios.

The objective of this work is to investigate the process conditions of a cooled finger device which is in operation for laboratory scale experiments (Curtolo et al. 2017), i.e. simulating solidification on the length scale of a few $\mathrm{cm}$ with pulling velocities up to $25 \mu \mathrm{ms}^{-1}$. We will consider the system layout as a Bridgemann type problem of directional solidification. In a first part, we will present results for constant temperature gradients $G$ between 1 and $10 \mathrm{Kcm}^{-1}$ and constant pulling velocities $V_{\text {pull }}$ up to $12 \mu \mathrm{ms}^{-1}$. This allow us to follow the microstructure evolution from the initial state to a quasi stationary growth situation which depends on the process parameters. In a second part, we will present simulation results for non-stationary temperature fields using an integrated macroscopic 1D-temperature solver. The temperature field solution is governed by heat fluxes at the system boundaries. This approach is closer to the real experimental process with temperature gradients up to $50 \mathrm{Kcm}^{-1}$ and solidification velocities up to $25 \mu \mathrm{ms}^{-1}$ and is described in detail for the first time. In particular, it takes into account the dynamical evolution of the temperature field at the start of the solidification. We have used a 2D phase field model, because dynamical phase field simulations can treat the problem of morphological stability and describe the segregation also for a cellular solidification front, whereas results from analytic 1D models are limited to a morphological stable planar front.

\section{Cooled finger}

The technical device is based on a rotating, internally gas cooled crystallization unit (so-called "cooled finger"), which is immersed into the melt as shown in Fig. 1. The initial aluminum melt temperature typically is around $700^{\circ} \mathrm{C}$. On the cooled finger sur- 


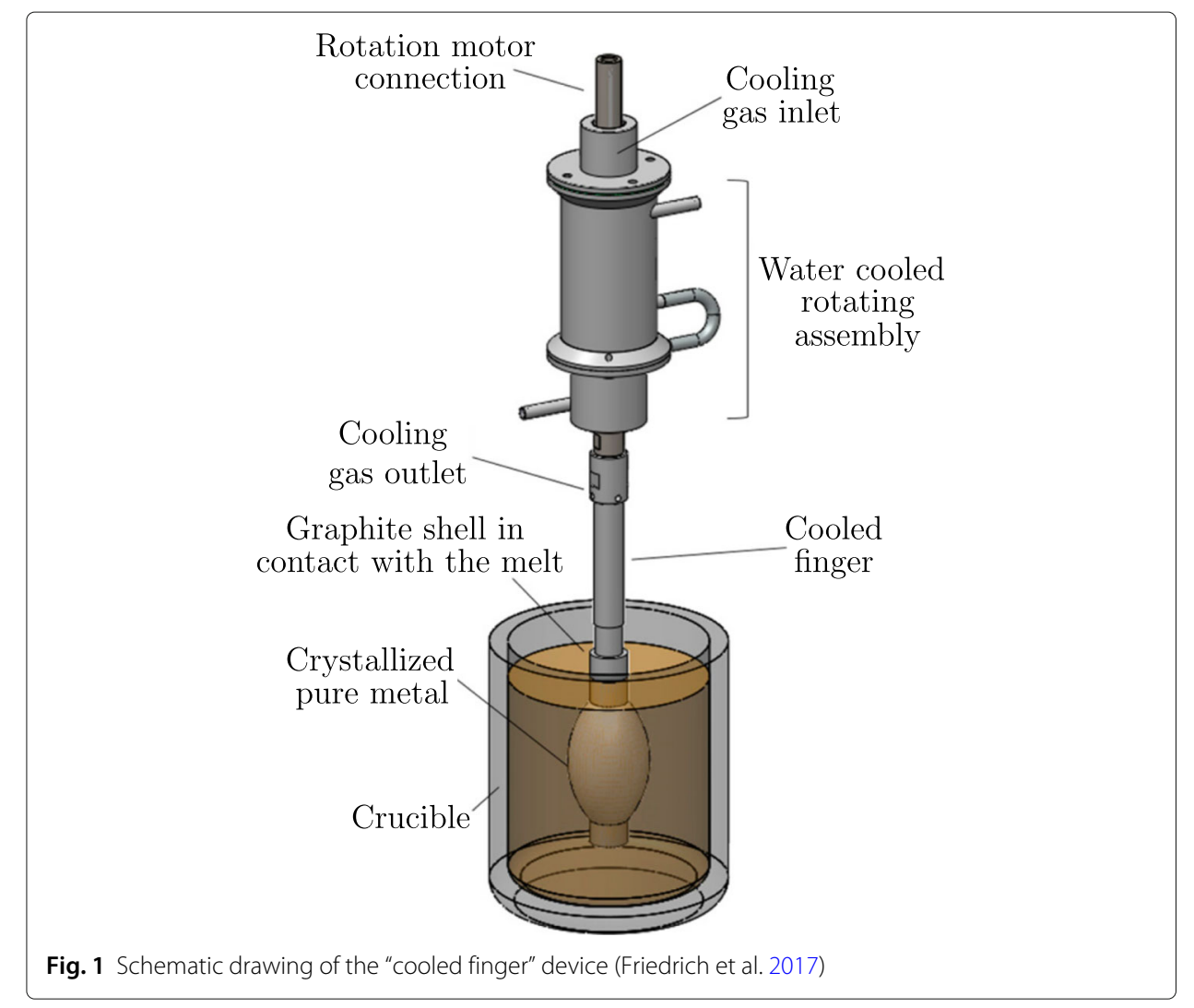

face, solidification will start when the temperature of the melt drops below the liquidus temperature. As already mentioned, the necessary requirement for purification is a morphologically stable, planar solidification front. For a cellular or dendritic solidification front morphology, impurities will be trapped in the intercellular or interdendritic liquid, leading to microsegregations in the solidified metal. The morphological stability of the growth front (Dantzig and Rappaz 2009) depends on the temperature gradient $G$ at the solidification front, the velocity of the solidification front $V$, the solidification interval $\Delta T_{0}$, the diffusion length $l_{D}$, and the diffusion coefficient in the melt $D_{l}$. The interface is morphologically stable if the relation for constitutional undercooling holds:

$$
\frac{G}{V} \geq \frac{\Delta T_{0}}{D_{l}} \text { or } G \geq \frac{V}{D_{l}} \cdot \Delta T_{0}=\frac{\Delta T_{0}}{l_{D}}
$$

It can be seen that a high temperature gradient and a low velocity of the solidification front have a stabilizing effect on the interface, a large solidification interval and small diffusivities have a destabilising effect on the interface. However, a high temperature gradient is associated with a large heat flow and would be energetically unfavorable, also low solidification velocities are unproductive due to long process times. In Fig. 2, a schematic plot of the concentration profile at the interface during solidification is shown. The rejected impurity elements are creating a solutal pileup at the interface with an exponential decay from the concentration at the interface $C_{L}(x)$ to the far field concentration in the melt $C_{L}$. The region represented by $\delta$ is the diffusion boundary layer. The melt in 


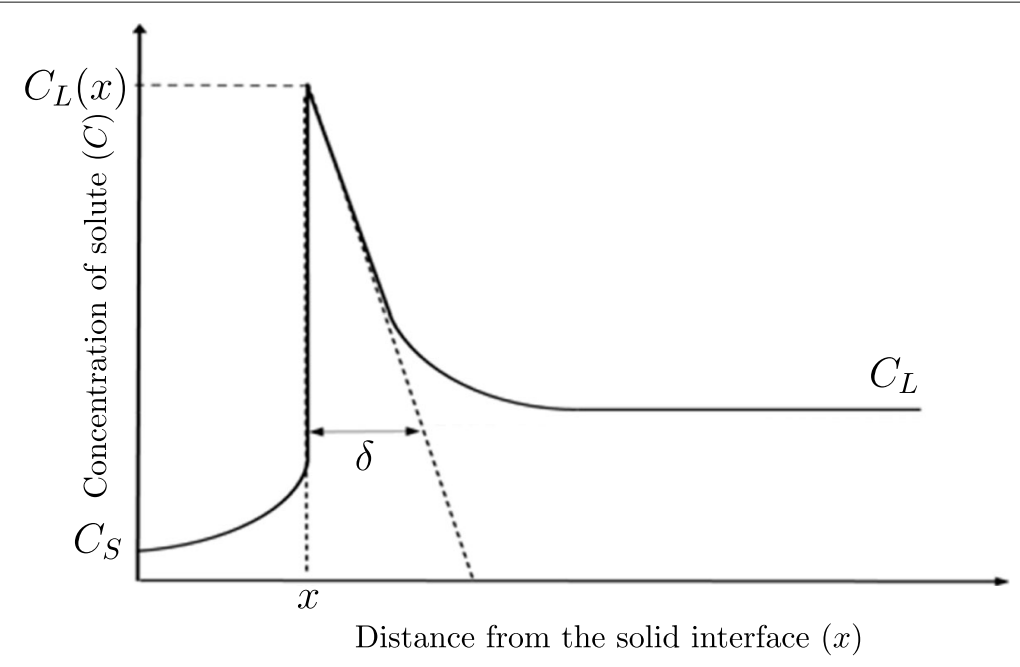

Fig. 2 Concentration pileup ahead of the solidification front (Friedrich et al. 2017)

this zone is in an undercooled state if the temperature gradient $G$ in the liquid at the interface is smaller than the gradient of the concentration dependent liquidus temperature in the solutal pile up which is the concentration gradient $\left(G_{c}\right)$ multiplied by the liquidus slope $m$ (Kurz and Fisher 1998).

$$
G<m G_{C}
$$

In this area, solute transport is diffusive, and ahead the transport is mainly governed by convection. For the cooled finger device, convection is mainly driven by the rotation of the cooled finger (Porter and Easterling 1992; Chatelain et al. 2015; Wilson 1978). Convection leads to a long range mixing of the melt and therefore decreases the solutal pileup thus stabilizing the planar interface even for smaller temperature gradients.

In the lab scale experiments, the thickness of the solidified material reaches a few centimeters, then the solidification is stopped by pulling the cooled finger out of the melt. Typical growth rates observed experimentally are between $1 \mu m s^{-1}$ to $25 \mu m s^{-1}$ (Curtolo et al. 2017).

\section{Modeling}

\section{Phase field model}

The simulations shown in this work are based on the multiphase field model linked to thermodynamic databases (Böttger et al. 2015; Eiken et al. 2006) as implemented in the phase field software MICRESS (2018). A finite-difference correction was used to improve the accuracy of the results (Eiken 2012). Furthermore, an anti-trapping current and mobility correction according to the thin interface limit is considered (Carré et al. 2013). This phase field model has already been used to study the evolution of a mushy zone in Al-alloys in more detail (Boussinot et al. 2020, Boussinot and Apel 2017). 


\section{Integrated 1D temperature solver}

For advanced coupling of microstructure simulation to macroscopic temperature problems on the process scale, a 1D-macro model has been integrated into the phase-field software (MICRESS 2018), allowing for direct coupling between the global heat flow and the local release of latent heat due to phase transformation (Böttger et al. 2009). Such coupling is especially strong in case of low temperature gradients like e. g. in technical casting processes, as has been demonstrated in (Böttger et al. 2009). The 1D-approximation is formulated in Cartesian, spherical or cylindrical coordinates and thus well adapted to the process geometry in most cases.

As shown in Fig. 3, the 1D-temperature field is aligned with the $\mathrm{z}$-coordinate of the $2 / 3 \mathrm{D}$-microstructure domain such that it fully overlaps and optionally further extends above the top and/or below the bottom boundary of the microstructure domain. In the part overlapping with the microstructure domain, latent heat is calculated using enthalpy and heat capacity data obtained online from the Calphad database, while otherwise tabulated data of $H(T)$ and $C_{p}(T)$ are used. For solving the 1D-thermal conduction equation, a fixed grid explicit approach is used which is based on the heat source method (Lewis et al. 1996) where the latent heat $\mathrm{dL}$ is released in each control volume $V$ :

$$
d T=\frac{1}{\bar{C}_{p}}\left(\frac{d^{2} T}{d x^{2}} d t-d L\right) \text { with } \bar{C}_{p}=\frac{1}{V} \int_{V} \sum_{\alpha} C_{p, \alpha} \phi_{\alpha} d V .
$$

The thermal conductivity $\lambda$ is approximated in the region of the microstructure domain by volume averaging as $\lambda=\sum_{\alpha} \lambda_{\alpha}$ and tabulated as $\lambda(T)$ otherwise. In general, the isothermal change of the enthalpy $d H\left(\phi_{\alpha}, c_{k}\right)$ due to changes of phase fractions $\phi_{\alpha}$ and composition $c_{k}$ define the latent heat $d L$ inside the volume $V$ :

$$
d L=d H\left(\phi_{\alpha}, c_{k}\right)=\frac{1}{V \bar{C}_{p}} \int_{V} \sum_{\alpha}\left(\phi_{\alpha} \sum_{k} \frac{\partial H_{\alpha}}{\partial c_{k}} d c_{k}+H_{\alpha} d \phi_{\alpha}\right) d V .
$$

Thus, $d L$ can be evaluated from the total change of the average enthalpy $H$ in the control volume $V$ if the temperature contribution is subtracted:

$$
d L=d H\left(\phi_{\alpha}, c_{k}\right)-\bar{C}_{p}^{*} d T^{*} .
$$

In an explicit solver scheme $\bar{C}_{p}^{*}$ and $d T^{*}$ correspond to the average heat capacity and the temperature change from the last time step. In the parts of the 1D-temperature field where no microstructure information is available, latent heat can be evaluated in the

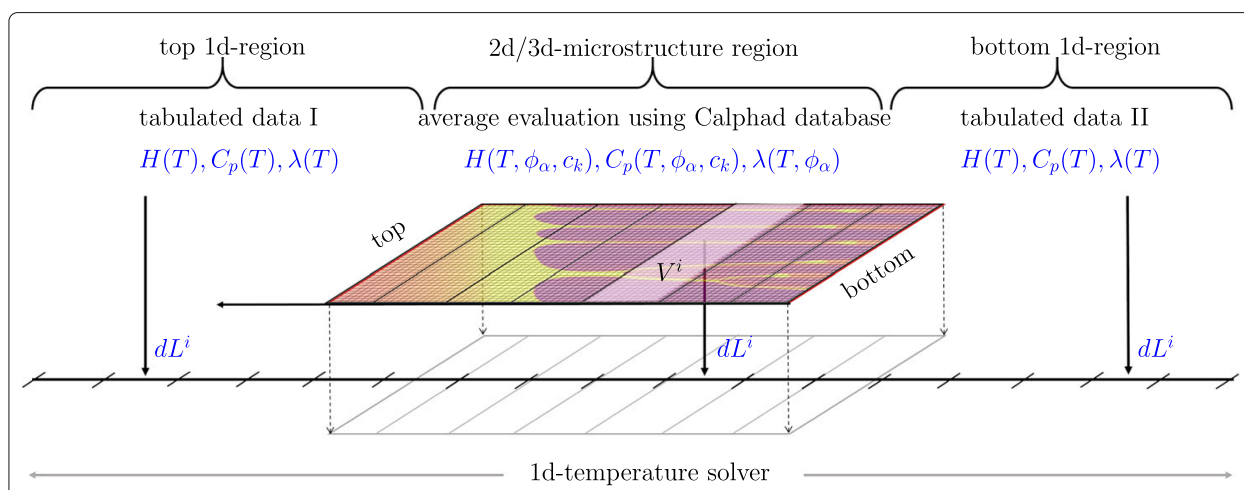

Fig. 3 Schematic view of the 1D-temperature solver and the calculation of latent heat in the different regions 
same way from tabulated data of $H(T)$ and $C_{p}(T)$. The described method for solving the temperature change in the simulation domain is quite simple, but has the disadvantage of introducing an additional stability criterion: if the enthalpy change with temperature $d H / d T$ is steep, fluctuations can occur because the latent heat attributed to a temperature change $d T$ is released instantaneously. Therefore, an artificial kinetic equation is introduced which slightly delays latent heat release. The effectively realized enthalpy change per time step is calculated as:

$$
d L^{e f f}=k \Delta L^{a c c}
$$

with $\Delta L^{a c c}$ being the locally accumulated enthalpy change which has not yet been released in the previous time steps. The kinetic constant $k=0.01$ is chosen such that latent heat release is not retarded significantly.

\section{Aluminum model alloy}

For our parametric study, we consider aluminum with the common impurity elements manganese, iron and silicon. The choice of the composition is rather arbitrary, but should be a step beyond a simple binary model towards technical metallurgical grade aluminum, e. g. from secondary sources. Of course, the efficiency of the purification, i. e. also the morphological stability of the solid/liquid interface, depends on the amount of impurities, however this will not be investigated further in this paper. The alloy composition is given in Table 1a. For this composition, the thermodynamic equilibrium calculation yields a liquidus temperature $\mathrm{T}_{l i q}=933.4 \mathrm{~K}$ and a solidus temperature $\mathrm{T}_{s o l}=931.5 \mathrm{~K}$, hence a solidification interval of $1.9 \mathrm{~K}$, whereas a Scheil-Gulliver calculation leads to a solidification interval of $4.9 \mathrm{~K}$. The thermodynamic calculations, also within the phase field simulations are based on Thermo-Calc using the database TCAL6. The segregation coefficients $k$ in Table 1a have been derived from a thermodynamic equilibrium calculation at $933 \mathrm{~K}$. The element partitioning in the phase field calculations are computed locally during runtime according to the quasi-equilibrium approach with a link to the thermodynamic database (Eiken et al. 2006). Hence, the segregation coefficients are not necessarily constant in the simulations, but due to the low impurity content, the conditions are those for a dilute solution with negligible variations of the segregation coefficients. The diffusion coefficients as used in the simulations are listed in Table $1 \mathrm{~b}$. The value for the interface energy of the solid/liquid interface is $2.5 \cdot 10^{-5} \mathrm{Jcm}^{-2}$ with $1.92 \%$ cubic anisotropy.

Table 1 Alloy composition and diffusion coefficients

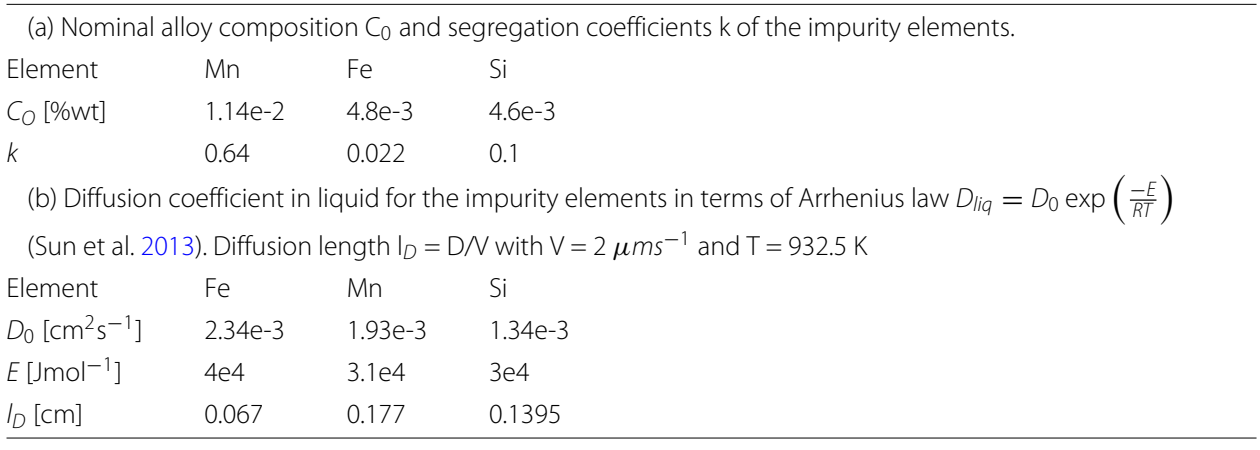




\section{Simulations with constant temperature gradient and pulling velocity Simulation parameters}

Simulations are performed in a rectangular domain of $1 \times 1.5 \mathrm{~mm}^{2}$, the simulated time period is $400 \mathrm{~s}$. Initially, a $1 \mathrm{~mm}$ thick solid fcc-Al layer with a planar interface is set at the bottom of the domain. The crystallographic orientation is rotated by $10^{\circ}$ from the z-direction which causes the asymmetry in the cellular growth pattern shown later (see Fig. 8). The initial layer circumvents the problem of nucleation. The initial bottom temperature was set to $\mathrm{T}_{0}=932.5 \mathrm{~K}, 0.9 \mathrm{~K}$ below the liquidus temperature. Hence, the initial interface is slightly undercooled as it would be also the case for nucleation of fcc-Al on the graphite cooled finger. Consequences will be discussed in more detail in the following. The boundary conditions are set to be periodic in $\mathrm{x}$-direction for concentration and phase field. For each impurity element a fixed concentration equal to its initial concentration specified in Table 1a is set on the top boundary. The computational domain moves with the solid/liquid interface to maintain a constant distance of $500 \mu \mathrm{m}$ between the most advanced point of the interface and the top boundary. This length can be considered as the diffusion boundary layer, and the Dirichlet condition at the top mimics perfect mixing of the far field melt. We studied a range of pulling velocities from $1 \mu \mathrm{ms}^{-1}$ to $12 \mu \mathrm{ms}^{-1}$ and temperature gradients from $1 \mathrm{Kcm}^{-1}$ to $10 \mathrm{Kcm}^{-1}$. Here, the pulling velocity is the constant velocity of the straight isotherms imposed by the predefined cooling rate and temperature gradient. The interface or growth velocity is the velocity of the evolving solid/liquid interface which is not constant at early stages but equal to the pulling velocity at steady state.

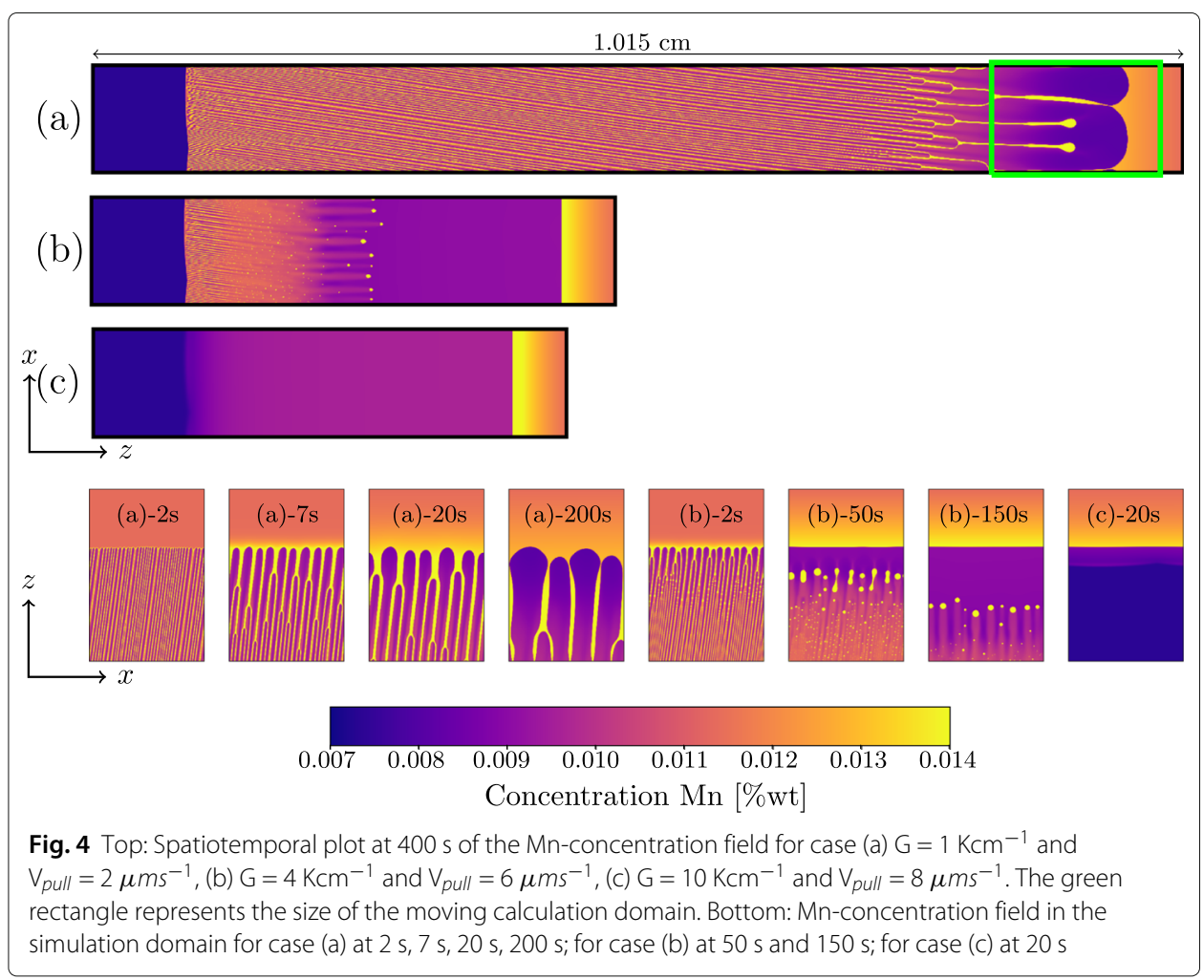




\section{The initial transient}

The transient stage of the microstructure evolution has already a crucial impact on the refining process if the growth conditions lead to a destabilization of the planar front. In the upper part of Fig. 4, we have plotted the spatiotemporal evolution of the Mnconcentration field for three different process parameter sets. The maps combine the moving computation domain (marked by the green rectangle) with the frozen concentration field which are moved out line by line at the bottom of the domain. Altogether, they represent the solidified aluminum after the first $400 \mathrm{~s}$ of growth. Remarkably, the final length of the spatiotemporal plot is more than $1 \mathrm{~cm}$ for case (a), although this is the case with the lowest steady state pulling speed, i. e. $\mathrm{V}_{\text {pull }}=2 \mu \mathrm{ms}^{-1}$ and $\mathrm{G}=1 \mathrm{Kcm}^{-1}$. Almost immediately, the planar interface evolves into a cellular morphology and remains cellular until the end of the simulation. As a consequence, there is no purification effect as the impurities are trapped within the intercellular channels, i. e. instead of a long range transport of impurities into the melt ("macrosegregation") only short range transport ("microsegregation") takes place. At the bottom of Fig. 4, the Mn-concentration in the calculation domain is shown at different times for the three process parameter sets.

The morphology for case (a) at $2 \mathrm{~s}$ is already cellular with an average cell spacing of $28 \mu \mathrm{m}$. At $7 \mathrm{~s}$, the growth velocity slows down and growth selection leads to an increasing cell spacing, but remains cellular until the end of the simulated time period. For case (b) with $\mathrm{G}=4 \mathrm{Kcm}^{-1}$ and $\mathrm{V}_{\text {pull }}=6 \mu \mathrm{ms}^{-1}$, the microstructure evolves from planar to cellular and becomes planar again. The cellular spacing for case (b) already starts increasing to an average spacing of approx. $45 \mu \mathrm{m}$ at $2 \mathrm{~s}$. At $50 \mathrm{~s}$, the growth front has become planar with a strong solute pileup in the liquid and remains planar for the rest period of time. The comparison of the composition in the solid and liquid in front of the pileup proofs the refinement, however growth is still transient as discussed later. For case (c) with $\mathrm{G}=10 \mathrm{Kcm}^{-1}$ and $\mathrm{V}_{\text {pull }}=8 \mu \mathrm{ms}^{-1}$, the solid/liquid interface remains planar over

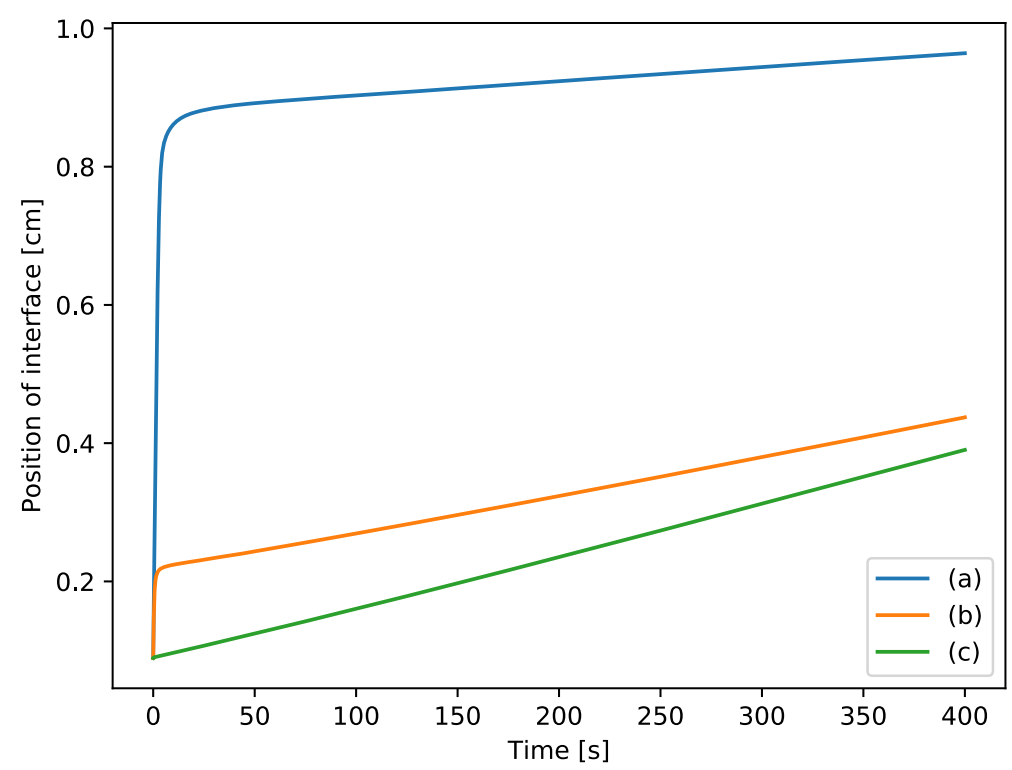

Fig. 5 Interface position as a function of time for case (a), (b) and (c) from Fig. 4 
the whole time. The concentration map shows an increasing Mn-concentration in the solidified aluminum.

In order to further investigate the initial transient, the position of the solid/liquid interface, i. e. the foremost advanced point in the simulation domain, is plotted over time in Fig. 5. In case (a), the interface velocity is approx. $1300 \mu \mathrm{ms}^{-1}$ during the first 6 seconds. After $20 \mathrm{~s}$, the interface velocity converges to the steady state pulling velocity of only $2 \mu \mathrm{ms}^{-1}$, which corresponds to the slope of the linear part after the kink. This behavior reflects the choice of the initial conditions. Initially, as mentioned above, the interface is off-equilibrium, at a temperature $\mathrm{T}_{0}^{i} \approx 0.9 \mathrm{~K}$ below the liquidus temperature $\mathrm{T}_{\text {liq }}$. This corresponds to a length

$$
l_{\text {trans }}=\frac{\left(T_{l i q}-T_{0}^{i}\right)}{G} \approx \frac{0.9 \mathrm{~K}}{1 \mathrm{Kcm}^{-1}}=0.9 \mathrm{~cm}
$$

for the growing interface until it reaches the equilibrium liquidus temperature. Because of the low impurity content, the growth restriction caused by solute diffusion is small, and the initial growth rate can be large, rather controlled thermally than by the solutal undercooling. The high velocity promotes the destabilization of the planar front. It is the same for case (b), but with a shorter "out of equilibrium" distance. For case (c), the initial interface position is already close to $\mathrm{T}_{\text {liq }}$ (please be aware that $\mathrm{T}_{0}$ is not exactly the initial interface temperature but the temperature at the bottom of the simulation domain) and thus, the interface velocity corresponds to the pulling velocity right from the beginning. The length of the initial growth period $l_{\text {trans }}$ is $7.9 \mathrm{~mm}, 1.1 \mathrm{~mm}$ and $0.2 \mathrm{~mm}$ for case (a), (b) and (c), respectively. These values agree well with the position of the kink in plot Fig. 5.

In Fig. 6, the normalized concentration profile of manganese along the z-axis is plotted for the three cases by averaging the concentration $C_{M n}(x, z)$ over x (see Fig. 4). One can see directly that during the fast initial growth in case (a) and (b), the normalized concentration oscillates around unity, indicating that no refinement takes place. When the growth front remains, case (c), or becomes, case (b), planar, the concentration profile is smooth and the concentration in the solidified aluminum is smaller than the initial concentration in the melt. The concentration profile in the liquid also interferes with the Dirichlet boundary condition at the top of the moving simulation domain, this will be further discussed in Effect of the boundary layer thickness section.

A central conclusion from the results so far is that the nucleation undercooling, i. e. the starting point for solidification, has a large impact on the refinement process. Even when the temperature gradient and growth velocity lead to a planar growth front in steady state, a large nucleation undercooling results in a cellular growth pattern in the first part of the solidified material with no refinement. This reduces the efficiency of the process with respect to purification, as the total fraction of purified aluminum is reduced. This is illustrated by the Fig. 4 where a large zone of cellular patterns is observed in case (a) and less in case (b).

\section{Comparison with analytic 1D models}

The steady state solution for a diffusion controlled planar solidification front is characterized by an exponential decay of the solutal pileup in the melt. Mass balance requires $\mathrm{c}_{s}=\mathrm{c}_{0}$ and thermodynamic equilibrium $\mathrm{c}_{s}=\mathrm{k}_{0} \cdot \mathrm{c}_{l}$ at the interface. The formation of this 


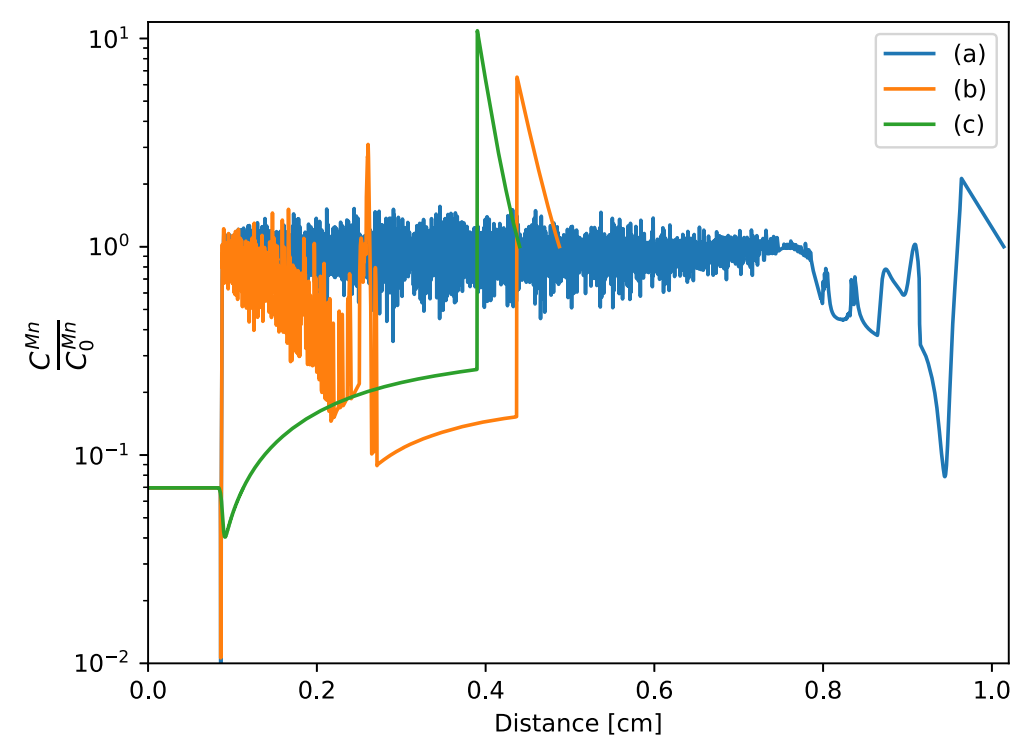

Fig. 6 Normalized Mn-concentration as a function of $z$ for case (a), (b) and (c) at $t=400$ s from Fig. 4, z=0 corresponds to the bottom of the simulation domain

pileup corresponds to the initial transient towards steady state. Along this initial transient, the concentration distribution in the solid can be described by (Dantzig and Rappaz 2009; Kurz and Fisher 1998), if the initial undercooling is neglected :

$$
c_{s}=c_{0}\left(1-\left(1-k_{0}\right) \exp \frac{-v k_{0}}{D} z\right) .
$$

The length corresponding to this initial period is determined by the diffusion length $\mathrm{v} / \mathrm{D}$ and the segregation coefficient $\mathrm{k}_{0}$. The equation shows that a diffusion limited planar front solution will only lead to purification during this initial period as $\mathrm{c}_{s}=\mathrm{c}_{0}$ for $z \longrightarrow \infty$. Hence, the solutal pileup has to be removed, e. g. by convection which is the major argument for rotating the cooled finger. The limiting case is perfect mixing of the liquid and therefore no concentration gradient ahead of the solid/liquid interface. This is described by the Scheil-Gulliver model (Kurz and Fisher 1998) with

$$
\begin{aligned}
c_{s} & =k_{0} c_{0}\left(1-f_{s}\right)^{k_{0}-1} \\
f_{s} & =\frac{z}{L_{z}} .
\end{aligned}
$$

The solidified fraction can be expressed by the length of solidified material $\mathrm{z}$ divided by the total length $\mathrm{L}_{z}$. In a closed system, the Scheil-Gulliver equation defines the best purification possible.

In Fig. 7, we have compared the solutions of Eqs. 7 and 8 with the concentration profiles from the phase field simulations. We can observe that for the case with $\mathrm{G}=1 \mathrm{Kcm}^{-1}$ and $\mathrm{V}_{\text {pull }}=2 \mu \mathrm{ms}^{-1}$ the concentration profiles from the simulations are far from the transient planar front predictions based on Eq. 7 because the initial stage of the transformation is highly cellular. For the case $\mathrm{G}=4 \mathrm{Kcm}^{-1}$ and $\mathrm{V}_{\text {pull }}=6 \mu \mathrm{ms}^{-1}$, in the first $\approx 0.25 \mathrm{~cm}$ the morphological evolution is also cellular, but the concentration in the solid decreases gradually when the front evolves towards planar growth. The concentrations tend to values between Gulliver-Scheil and initial transient predictions. For the third case, 


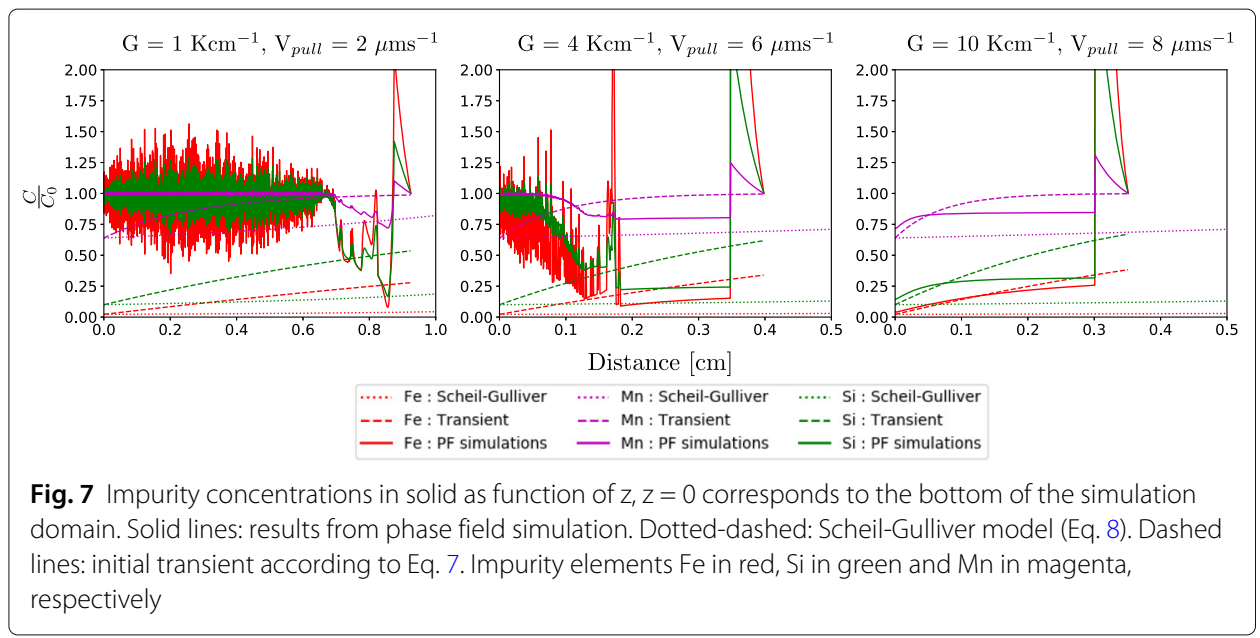

$\mathrm{G}=10 \mathrm{Kcm}^{-1}$ and $\mathrm{V}_{\text {pull }}=8 \mu \mathrm{ms}^{-1}$, the interface remains planar right from the beginning. Initially, the concentrations are slightly above the transient solutions, which is due to the initial undercooling not considered in Eq. (7). But eventually the concentrations reach stationary values between $c / c_{0}=1$ and the values from Gulliver-Scheil. This is an indication that the solutal pileup interferes with the boundary condition, i. e. the diffusion length is of the same order as the length of the diffusion boundary layer. This aspect will be further discussed in Effect of the boundary layer thickness section.

\section{Steady state morphologies}

In Figs. 8, 9, a morphology diagram is shown with concentration maps obtained at $400 \mathrm{~s}$ for different temperature gradients and pulling velocities. The solidification front morphology is represented on a diagram with increasing velocities on the horizontal axis and increasing temperature gradients on the vertical axis. The diagram shows a transition from planar to cells with decreasing temperature gradients and increasing pulling velocities. In fact a high temperature gradient $>8 \mathrm{Kcm}^{-1}$ and low front velocity $<4 \mu \mathrm{ms}^{-1}$ promotes planar growth, but is less efficient in terms of energy consumption and processing times for a technical refinement process. For the alloy composition in this case study, a temperature gradient of $8 \mathrm{Kcm}^{-1}$ would require a velocity below $9 \mu \mathrm{ms}^{-1}$ for the planar growth.

In order to evaluate the efficiency of the refinement for the different combinations of $\mathrm{V}_{\text {pull }}$ and $\mathrm{G}$ including the transient behavior at the beginning, the concentration fields in the spatiotemporal domains, e. g. shown in Fig. 6, are averaged over the solidified portion. The data from all parameter sets are compiled in the 3D plot shown in Fig. 10. The 3D plane has a "cuvette"-like shape, showing the best refining efficiency for low front velocities and large temperature gradients. The steep increase with higher velocities $>8 \mu \mathrm{ms}^{-1}$ reflects the transition from planar to cellular steady state morphologies. The smoother increase with decreasing gradients reflects also the impact of the initially cellular growth before the front becomes planar and the solidification velocity reaches its steady state.

\section{Effect of the boundary layer thickness}

Ahead of the diffusive boundary layer, convection adds to the impurity transport in the melt. As already mentioned, long range transport by melt convection is essential for the 


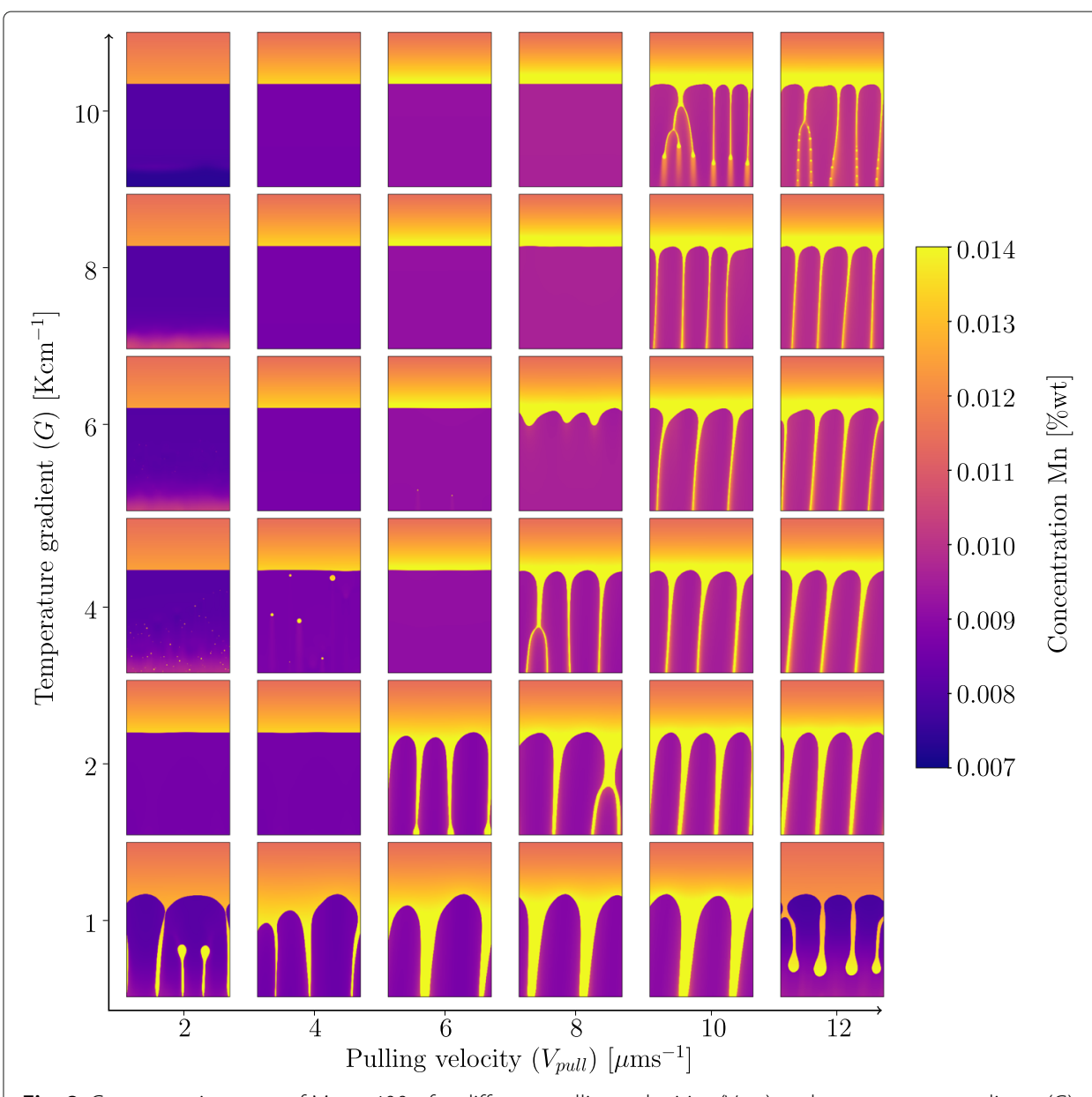

Fig. 8 Concentration map of $\mathrm{Mn}$ at $400 \mathrm{~s}$ for different pulling velocities $\left(V_{\text {pull }}\right)$ and temperature gradients $(G)$

refinement in the cooled finger process. An accelerated impurity transport is equivalent to a larger effective diffusion length $l_{D}$ and according to Eq. 1 this improves the morphological stability, i. e. allows a stable solidification front for lower thermal gradients G. To investigate the effect of the boundary layer thickness in the phase field simulations, we have varied the moving frame distance, i. e. the distance between the interface and the

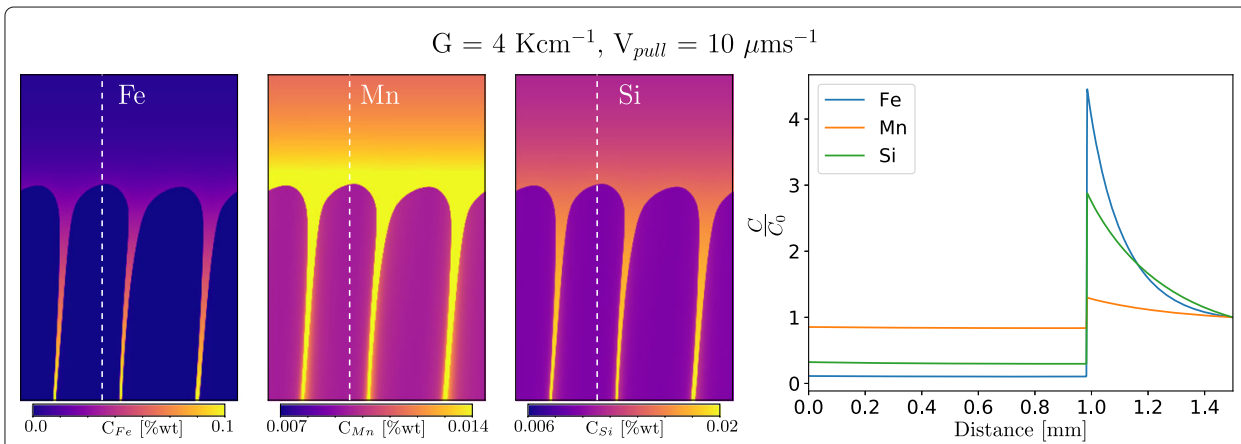

Fig. 9 (left) Concentration maps of Fe, Mn, Si at $400 \mathrm{~s}$ for the pulling velocity $\left(\mathrm{V}_{\text {pull }}\right)$ of $10 \mu \mathrm{ms}^{-1}$ and temperature gradient $(G)$ of $4 \mathrm{Kcm}^{-1}$. (right) Concentration profiles for each element along the white dashed line 


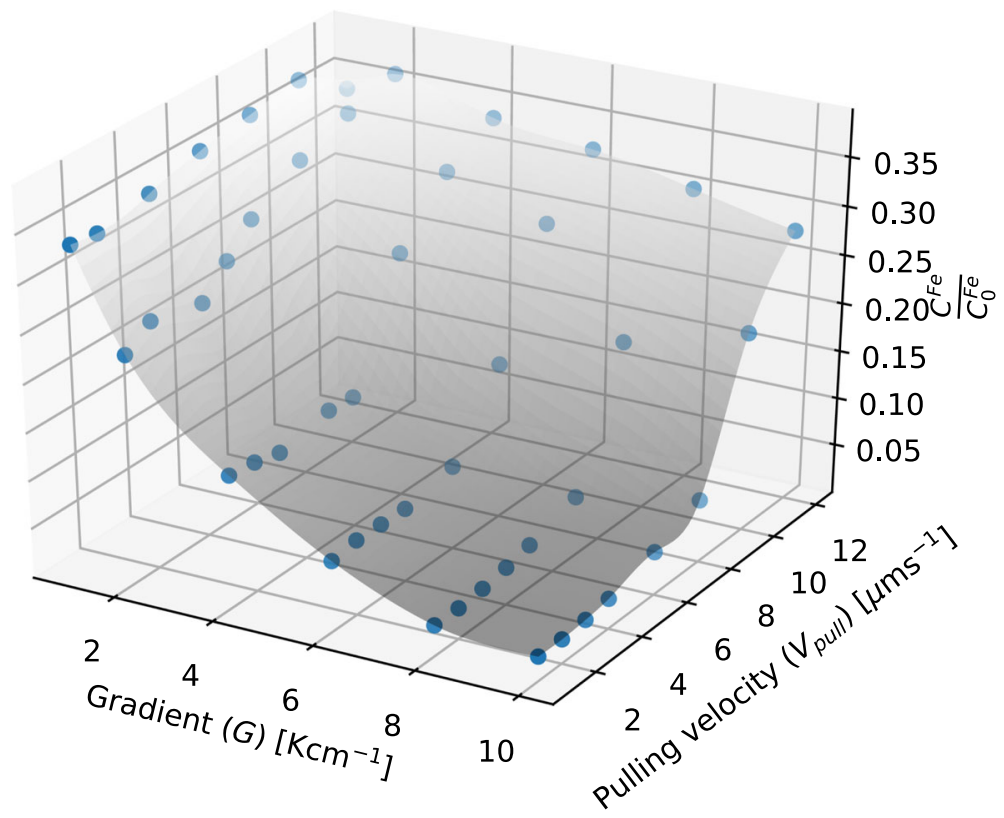

Fig. 10 Averaged Fe-concentration within the solidified material in the first $400 \mathrm{~s}$ of growth as a function of temperature gradient $\mathrm{G}$ and pulling velocity $\mathrm{V}_{\text {pull }}$

top boundary of the moving simulation domain. Results are shown in Fig. 11. Here, steady state concentration profiles are plotted for different moving frame distances.

With decreasing moving frame distance, the maximum concentration at the solid/liquid interface decreases and, because of $\mathrm{c}_{s}=\mathrm{k} \cdot \mathrm{c}_{l}$, the concentration in the solid decreases as well, leading to a better refinement. In practice, the effective boundary layer thickness can

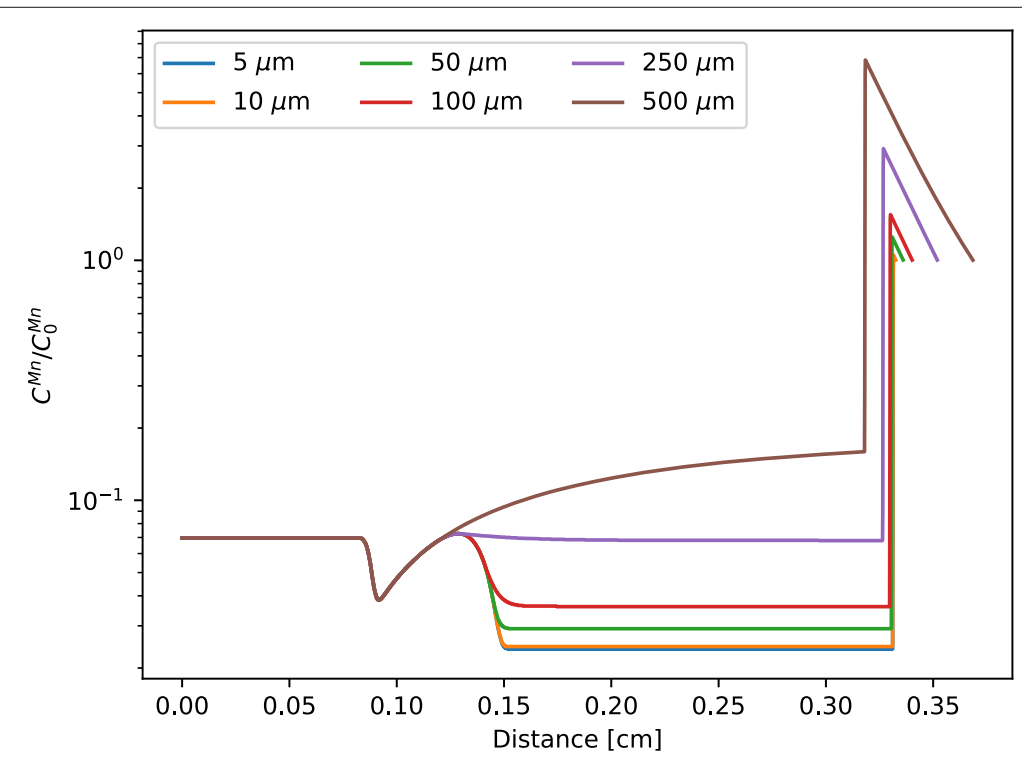

Fig. $11 \mathrm{Mn}$-concentration profiles for different moving frame distances. Simulations with $\mathrm{G}=10 \mathrm{Kcm}^{-1}$ and $V_{\text {pull }}=6 \mu \mathrm{ms}^{-1}$ 
be controlled by the rotation speed of the cooled finger. It has to be lower than the diffusion length to have an effect on the interfacial pileup. An optimum refinement according to Gulliver-Scheil would require $\delta \longrightarrow 0$.

\section{Energy consumption}

The energy efficiency of the process can be estimated by the heat flux through the cooling finger device. The heat flux $\lambda_{s} \cdot G$ multiplied by the surface $S$ equals to the necessary power $P$ to maintain the thermal gradient.

$$
P=-\lambda_{S} S G
$$

$-\lambda_{S}$ is the solid thermal conductivity already defined in "Simulation parameters" section and equal to $\lambda_{s}=1.9 \mathrm{Wcm}^{-1} K^{-1}$. For $S$, a cylindrical surface in the middle of the crucible can be used for the approximation. For the lab scale device shown in 1 we obtain $S=$ $2 \pi r \cdot h$ with radius $r=10 \mathrm{~cm}$ and height $h=18 \mathrm{~cm}$. The process time $t$ is the thickness of the solidified cylindrical shell divided by the pulling velocity, hence the total energy consumption $\mathrm{Q}$ is

$$
Q=P \cdot t=\lambda_{S} G 2 \pi r^{2} h / V_{\text {pull }}
$$

The plot in Fig. 12 shows the energy consumption depending on temperature gradient and pulling speed. For the best refining parameters (high gradient and low velocity, point 6 in Fig. 12), the energy needed is around $300 \mathrm{kWh}$. For other parameter sets (point 1 to 5) where the interface is also planar at the end of transformation (see Fig. 8), the energy consumption can be reduced by a factor of 5 to 10 on the cost of the average purity of the refined aluminum. Here, the amount of residual $\mathrm{Fe}, \mathrm{Si}$ and $\mathrm{Mn}$ is higher compared to the process conditions belonging to point 6 (see Fig. 10).

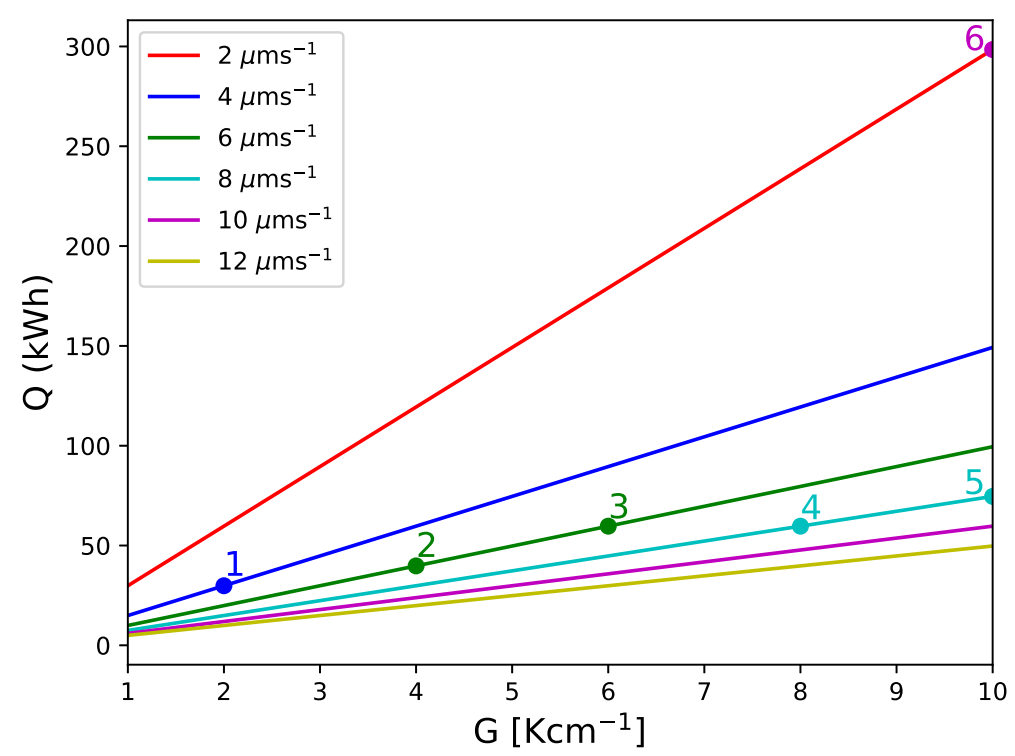

Fig. 12 Estimated energy consumption $Q$ depending on growth velocity $v$ and thermal gradient $G$. The process parameters according to Eq. 9 are indicated by the numbered points 


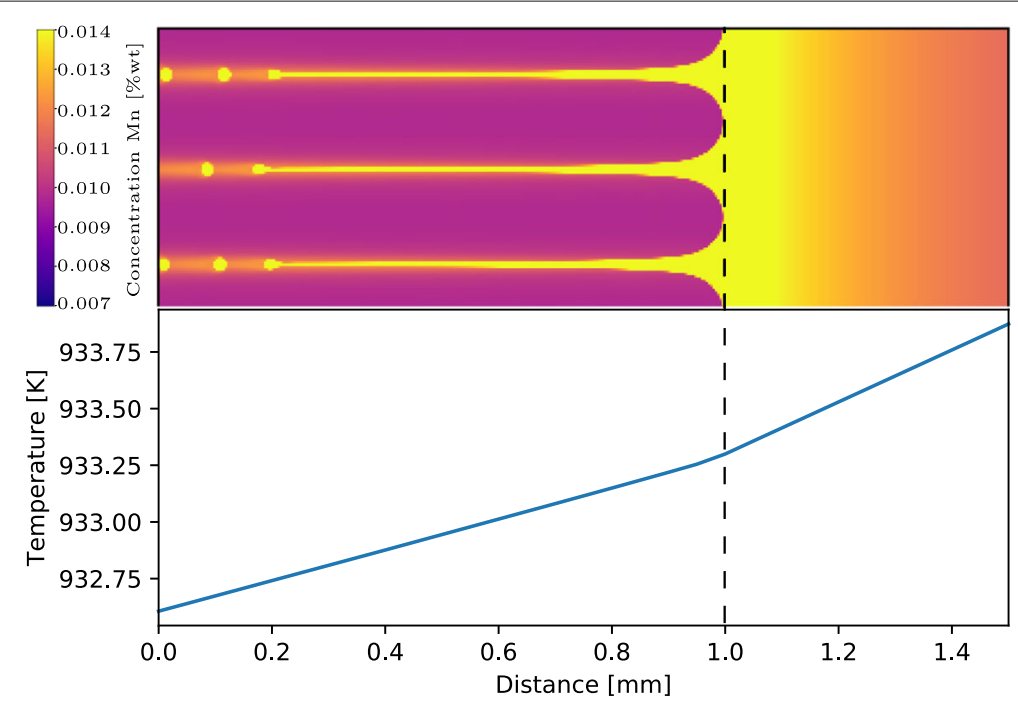

Fig. $13 \mathrm{Mn}$-concentration map (top) and temperature profile (bottom) for a state with $\langle G\rangle=8.3 \mathrm{Kcm}^{-1}$ and $V_{\text {front }}=11.4 \mu \mathrm{ms}^{-1}$

\section{Simulations with a dynamical temperature field}

\section{Simulation parameters}

In the technical process, the temperature field is controlled by the heat removal via the cooled finger and by the heater at the outside of the crucible. Therefore, the temperature field evolves dynamically over time depending on the cooling and heating rates and the latent heat release from solidification. In order to approximate the temperature evolution on the scale of the device, the approach presented in "Integrated 1D temperature solver" section has been used. The 1-dimensional macroscopic temperature field has a total length of $10 \mathrm{~cm}$, corresponding to the geometry of the experimental device in Fig. 1. The microstructure simulation domain had again a size of $0.5 \times 1.5 \mathrm{~mm}^{2}$ with a grid resolution of $\Delta \mathrm{x}=1 \mu \mathrm{m}$. Initially, the simulation domain is aligned to the bottom of the 1D-temperature field, which corresponds to the graphite surface of the cooled finger. By applying a co-moving frame, the domain was allowed to follow the solidification front with a fixed distance of $0.5 \mathrm{~mm}$ between the solid/liquid interface and the upper domain boundary. Therefore, it is a similar simulation as before, with the difference that the temperature field is now computed by the $1 \mathrm{D}$ approximation with a coupling between latent heat release from the moving solidification front and the temperature field calculations. In this setting, the solidification velocity and temperature gradients can be determined indirectly via the heat flux densities $j_{T}$ and $j_{B}$ at the bottom and top boundary of the 1D-temperature field, respectively, see Fig. 3. While the steady state solidification front velocity $V_{\text {front }}$ can be directly calculated from the energy balance between heat flux densities and latent heat $L$ per volume $L=1070 \mathrm{Jcm}^{-3}$ (Thermocalc 2019)

$$
V_{\text {front }} \cdot L=j_{B}-j_{T},
$$

the average temperature gradient $\langle G\rangle$ cannot be determined in a simple way. This is because the heat conductivity of the liquid and solid phase are significantly different $\left(\lambda_{s}=1.9 \mathrm{Wcm}^{-1} K^{-1}, \lambda_{l}=1.0 \mathrm{Wcm}^{-1} K^{-1}\right)$, and the region where latent heat is released 


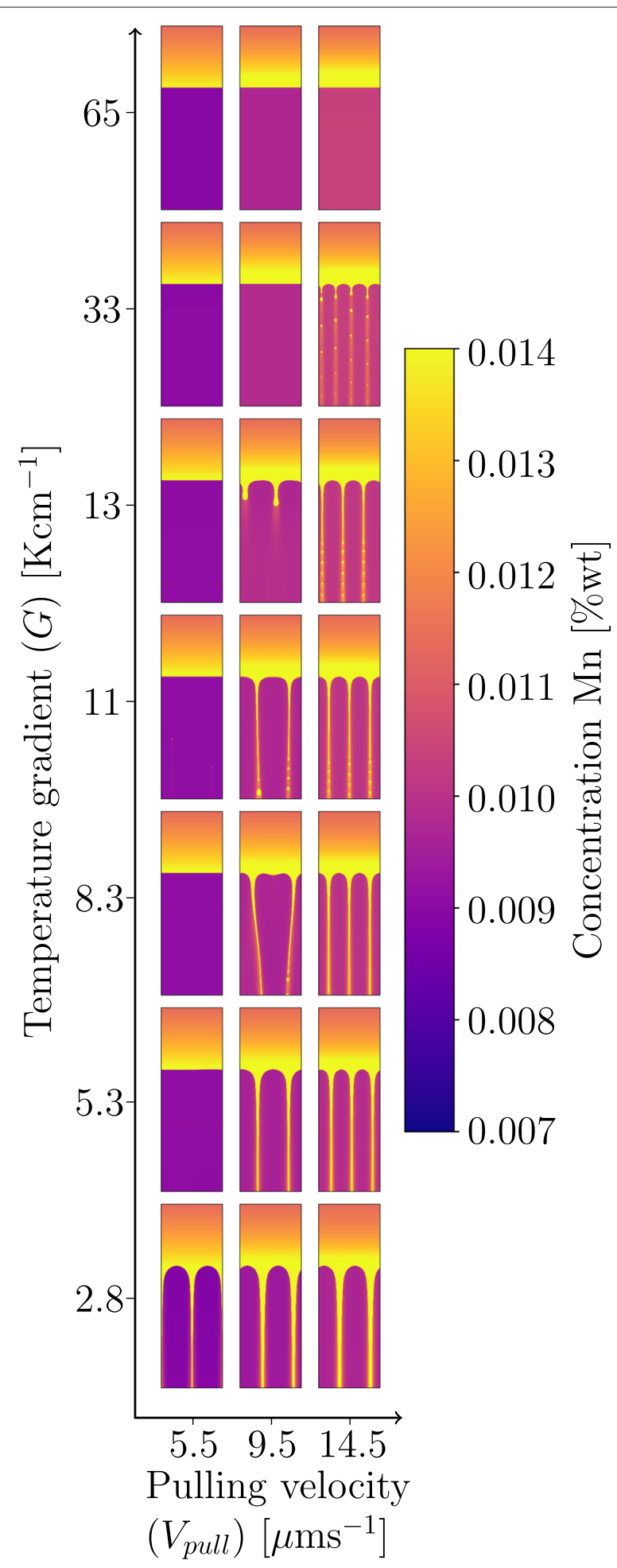

Fig. 14 Growth pattern (Mn-concentration) at $\mathrm{t}=400 \mathrm{~s}$, simulated with 1D temperature field calculations. Results are arranged according to the front velocities $V_{\text {front }}$ and average temperature gradients $\langle G\rangle$ 


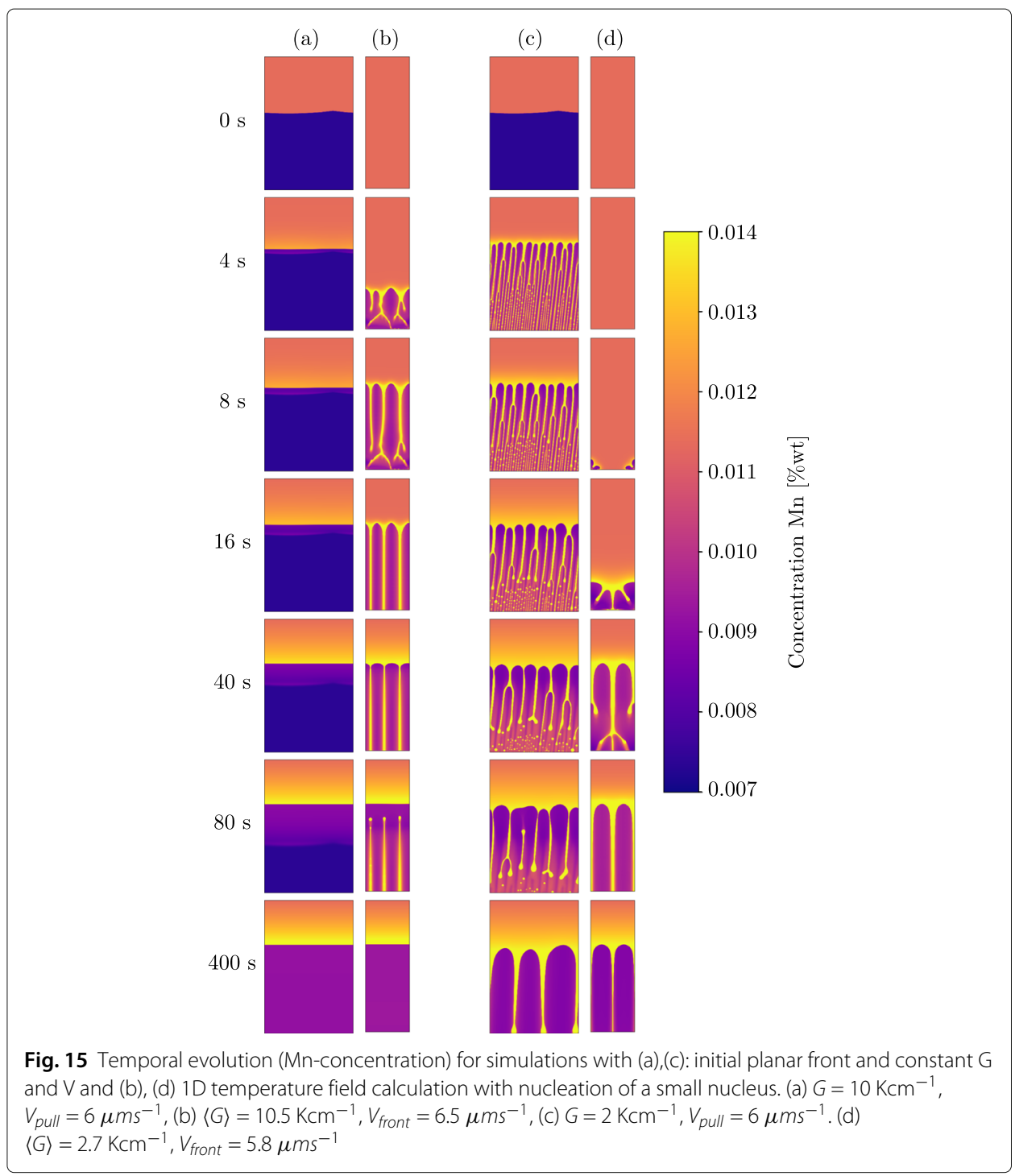

depends on the microstructure, see Fig. 13. Thus, to compare with the results obtained from the simulations with constant temperature gradient and pulling speed, the average gradient was obtained individually from each simulation run for the last time step close to stationary growth. The corresponding steady state morphologies are plotted in Fig. 14.

Instead of starting with a planar interface like in "Steady state morphologies" section, solidification is here initiated by a small nucleus of size $r=\Delta x$ at the bottom of the domain when a local melt undercooling of $2 \mathrm{~K}$ is reached. The preferential crystallographic growth orientation of the nucleus is aligned to the $\mathrm{z}$-direction.

\section{Interface morphologies}

Figure 14 shows a morphology diagram for the solidification pattern at $400 \mathrm{~s}$ for the simulations with the 1D temperature field calculation. The simulation conditions have been varied by selecting different values for $\mathbf{j}_{B}$ and $\mathbf{j}_{T}$. The interface morphologies are arranged in the diagram with the growth front velocity $V_{\text {front }}$ on the horizontal axis and the average 


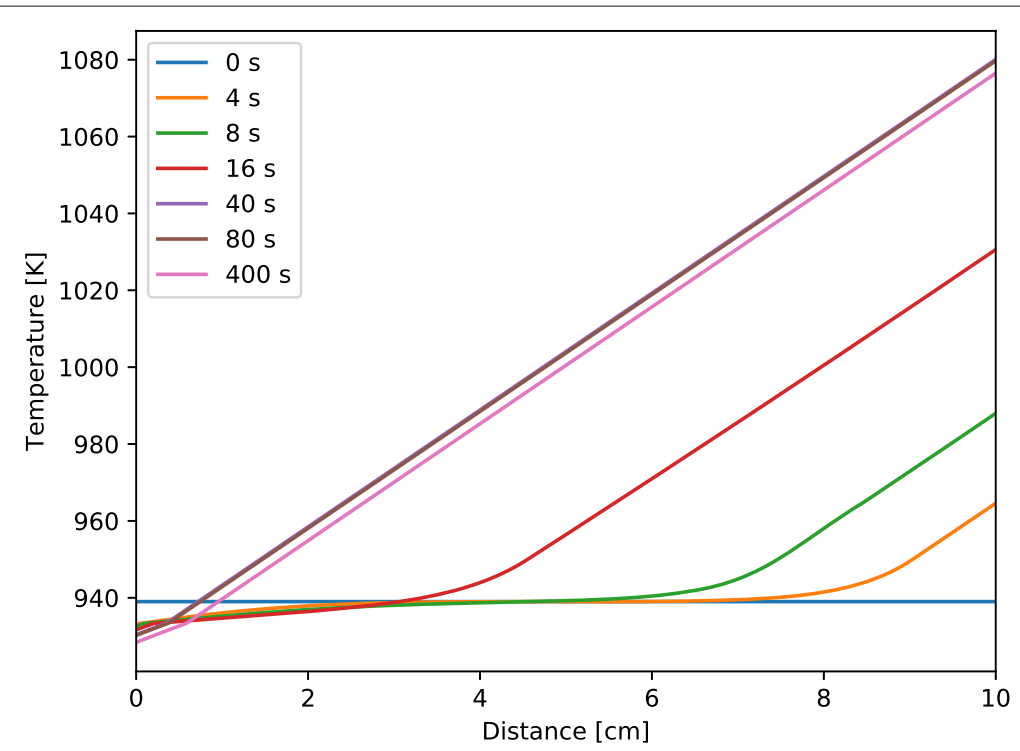

Fig. 16 Evolution of the temperature field for case (b) in Fig. 15

temperature gradient $\langle G\rangle$ on the vertical axis, similar to Fig. 8. It shows the demarcation between planar and cellular growth pattern with decreasing $G$ and increasing $V_{\text {front }}$.

In comparison to simulations with constant $\mathrm{G}$ and $\mathrm{V}$, the temporal evolution of the growth front morphology is represented in Fig. 15. The growth sequences compare simulations with similar $\mathrm{G}$ and $\mathrm{v}$ values, with and without $1 \mathrm{D}$ temperature field calculations, which results in either a planar or a cellular growth front. The values for (a), constant gradient $G=10 \mathrm{Kcm}^{-1}$ and constant velocity $V_{\text {pull }}=6 \mu \mathrm{ms}^{-1}$, and for (b), 1D temperature field, $\langle G\rangle=10.5 \mathrm{Kcm}^{-1}$ and $V_{\text {front }}=6.5 \mu \mathrm{ms}^{-1}$ lead both to a planar growth front, the combinations (c), constant gradient $G=2 \mathrm{Kcm}^{-1}$ and constant $V_{\text {pull }}=6 \mu \mathrm{ms}^{-1}$ and (d), 1D temperature field, $\langle G\rangle=2.7 \mathrm{Kcm}^{-1}$ and $V_{\text {front }}=5.8 \mu \mathrm{ms}^{-1}$ to cellular growth.

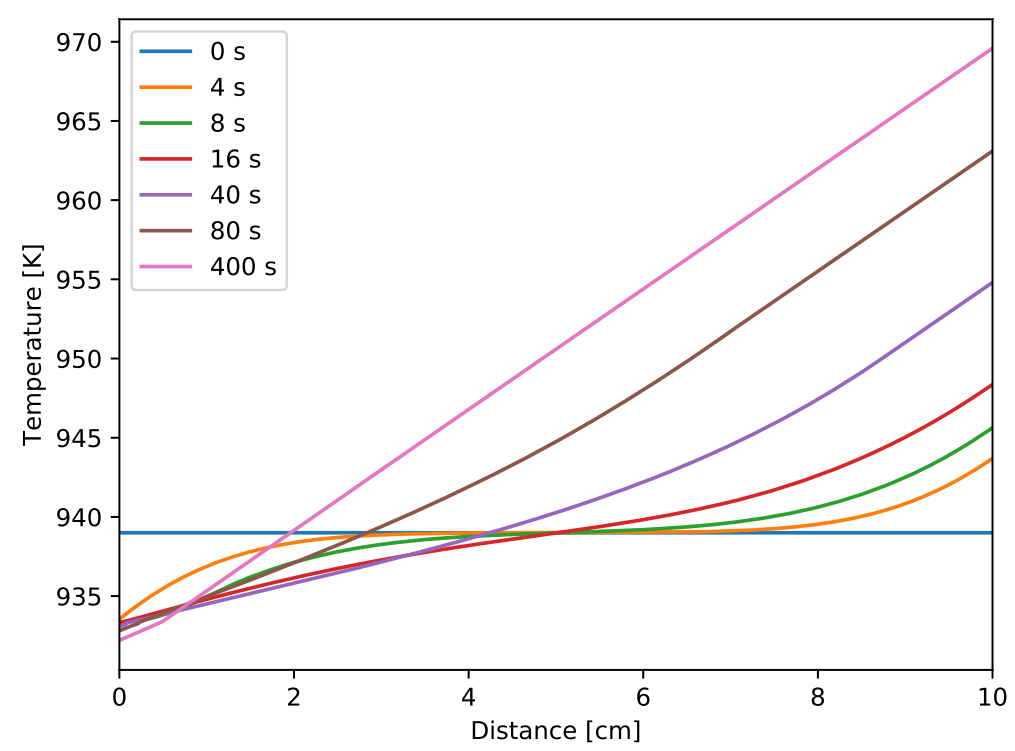

Fig. 17 Evolution of the temperature field for case (d) in Fig. 15 
The corresponding temperature fields for the simulations (b) and (d) are plotted in Fig. 16 and Fig. 17. Heating on the right side, which is the upper domain boundary corresponding to the crucible, leads to an increase of the initially constant melt temperature. Cooling on the left side, the position of the cooled finger, decreases the local melt temperature until the nucleation undercooling is reached. Then, fcc-Al nucleates and solidification starts. The latent heat release from solidification fixes the temperature in the solid/liquid mushy region to the interval [ $\left.T_{\text {sol }}, T_{\text {liq }}\right]$, see Fig. 13 . Therefore, the position of the growth front at later times can be identified by a kink in the temperature profiles .

The position of the interface for the four cases is plotted in Fig. 18. The initial position is different for case (a) and (c) than for (b) and (d) as one can directly see in Fig. 15. Simulations (a) and (c) start with a planar, preexisting solid layer with a thickness around $800 \mu \mathrm{m}$, (b) and (d) with nucleation at the domain bottom. In case (a), the interface reaches a stationary growth velocity with a morphologically stable planar front after a few seconds. In case (b), there is a transient stage of $\approx 30 \mathrm{~s}$ where the microstructure evolves from cellular to planar. For the cases (c) and (d), the microstructure evolves either from planar into the cellular steady state morphology or remains cellular right from the beginning with a transient time around $150 \mathrm{~s}$. The evolution of the cellular growth front in Fig. 15 shows for (c) spacing selection, starting with a fine cellular spacing during the fast initial growth and ending up with deep cells with a spacing of $333 \mu \mathrm{m}$.

The morphologies of the solidification front $400 \mathrm{~s}$ after start of the process have been compiled in Fig. 19 as a kind of morphology diagram sorted by $G$ and $V_{\text {pull }}$. We like to emphasize that the growth patterns at this stage are not necessarily steady state morphologies but those at a certain point of time during the initial stage of the cooled finger process. Hence, these morphologies may still reflect the process history. In addition, the results are obtained for "moving frame" simulations with a finite distance $\delta$ between the solidification front and the domain boundary, and at the boundary the composition has been fixed to the initial impurity composition. This means that the ratio between $\delta$ and

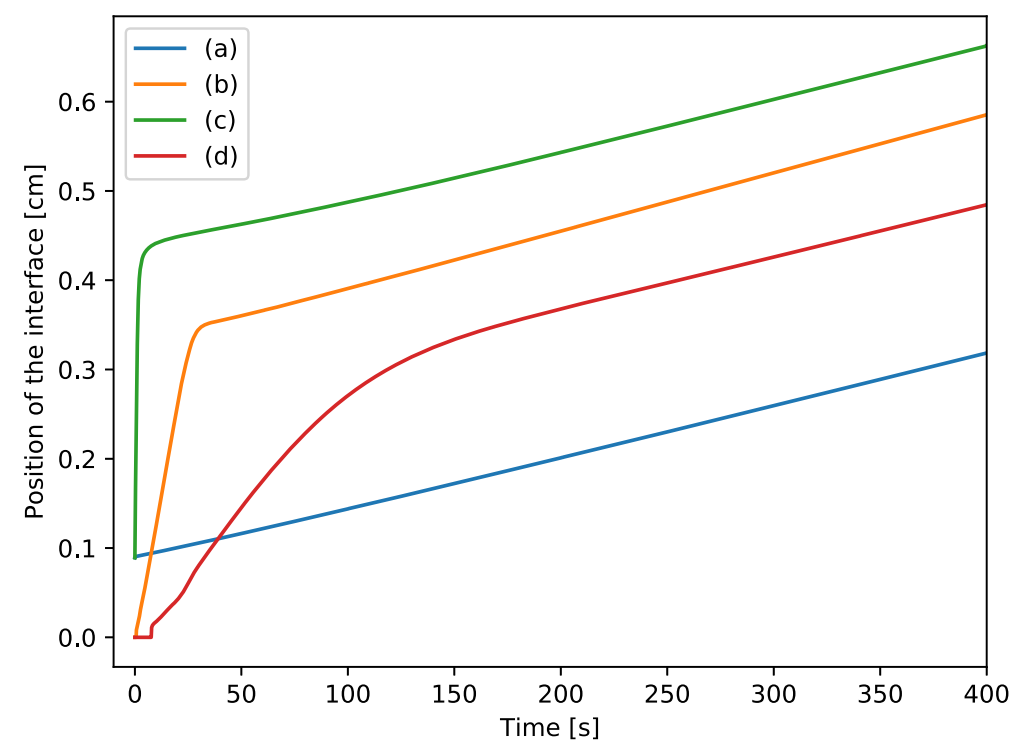

Fig. 18 Evolution of interface position with time for case (a), (b), (c), (d); for lettering see Fig. 15 


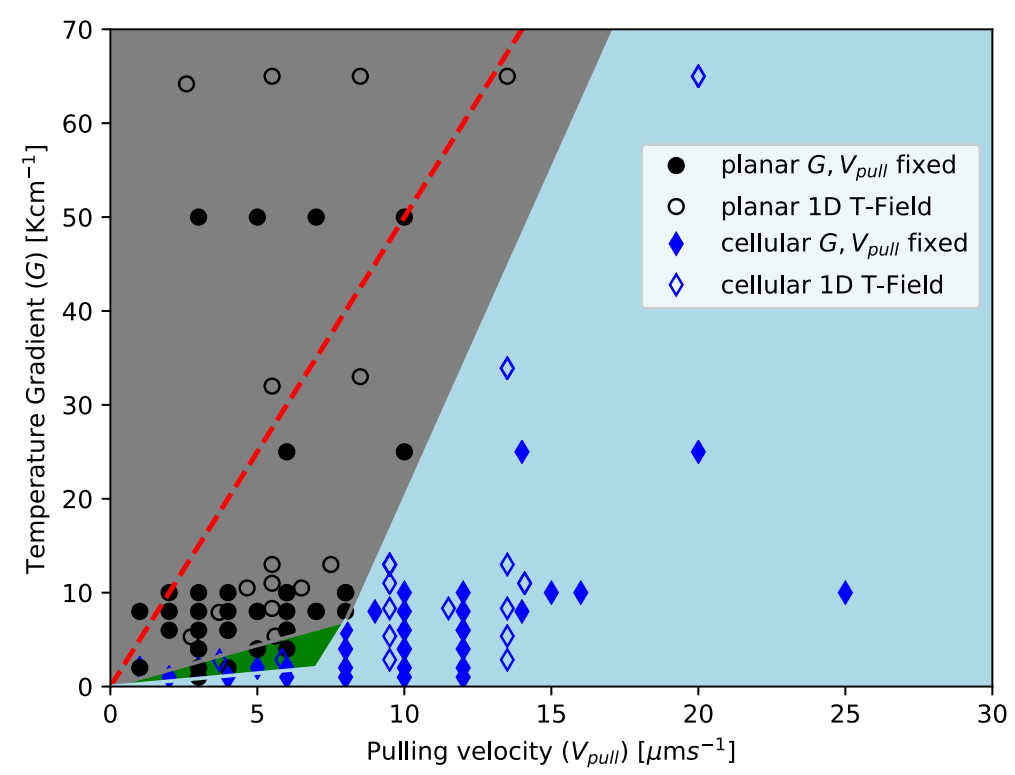

Fig. 19 Solidification front morphologies $400 \mathrm{~s}$ after start of solidification. The dashed red line indicates the constitutional undercooling criteria according to Eq. 1. The grey area indicates a morphologically stable planar front, the blue area of cellular growth morphologies and the green area the coexistence of cellular and planar morphologies

the diffusion length $D / v$ varies for the different growth velocities which has an impact on the morphological stability, the duration of the initial stage and thus on the demarcation between planar and cellular.

As a whole, the transition between planar and cellular morphologies is clearly visible in Fig. 19. The dashed red line is derived from the constitutional undercooling criteria according to Eq. 1, using a solidification interval of $2 \mathrm{~K}$ and a diffusion coefficient of $4 \cdot 10^{-5} \mathrm{cms}^{-1}$. It roughly approximates the planar/cellular transition for our dynamical multicomponent simulations. However, for lower values of G and V, e. g. $V_{\text {pull }} \leq 10 \mu \mathrm{ms}^{-1}$ planar morphologies emerge beyond the stability line which is an indication of the stabilizing effect when the length of the pileup is in the order of $\delta$ and interferes with the upper domain boundary.

\section{Conclusion}

In this paper, we demonstrate how phase field simulations can be used to investigate a solidification based refinement process for aluminum on experimental length and time scales by combining microstructure simulations on a mesoscopic scale with a macroscopic temperature field calculation using a 1D approximation. We have applied the model to investigate the initial growth period of the so-called "cooled finger" process, i. e. the first $400 \mathrm{~s}$ of solidification. The solidification front morphology has been studied depending on the process parameters "thermal gradient" and "solidification velocity". Aluminium with the impurity elements Fe, Si and Mn has been used for the example case study.

The results demonstrate the impact of the initial transient on the refinement, hence on the process efficiency. The nucleation undercooling leads to larger initial growth velocities compared to the final steady state and thus may lead to a morphological instability 
in the early stages, in particular to cellular growth which prevents the refinement. Phase field simulations now support the identification of process conditions for a stable planar growth front which is a necessary condition for the refinement. The morphological stability criteria from solidification theory suggests large temperature gradients and low solidification velocities for a planar front, however, both conditions are inefficient in terms of energy consumption and process time. Therefore, phase field assisted process simulations in future could propose a time dependent adjustment of the heating and cooling power at the outer rim of the crucible and the cooled finger for time and energy efficient processing schemes, considering real multicomponent alloy compositions. This offers the opportunity to identify the limits for stable growth even for transient conditions. A time dependent process control is of special value, in particular to prevent cellular growth pattern in the early stages of the process.

Acknowledgments

Not applicable.

\section{Authors' contributions}

All authors designed the study, performed the simulations and data analysis and also contributed to the writing of the manuscript. The authors read and approved the final manuscript.

\section{Funding}

This work has been funded by the Deutsche Forschungsgemeinschaft (DFG), project number 421743304. Open Access funding enabled and organized by Projekt DEAL.

Availability of data and materials

The data that support the findings of this study are available from the corresponding authors on reasonable request.

\section{Declarations}

\section{Competing interests}

The authors declare that they have no competing interests.

Received: 11 August 2021 Accepted: 8 February 2022

Published online: 07 March 2022

References

D. C. Curtolo, N. Xiong, S. Friedrich, B. Friedrich, High- and ultra-high-purity aluminum, a review on technical production methodologies. Metals. 1407(11) (2021). https://doi.org/10.3390/met11091407

B. Böttger, J. Eiken, M. Apel, Phase-field simulation of microstructure formation in technical castings - a self-consistent homoenthalpic approach to the micro-macro problem. J. Comp. Phys. 228, 6784-6795 (2009). https://doi.org/10. 1016/j.jcp.2009.06.028

Böttger, B., J. Eiken, M. Apel, Multi-ternary extrapolation scheme for efficient coupling of thermodynamic data to a multi-phase-field model. Comp. Mat. Sci. 108, 282-292 (2015)

G. Boussinot, M. Apel, Phase field and analytical study of mushy zone solidification in a static thermal gradient: From dendrites to planar front. Acta Mater. 122, 310-321 (2017)

G. Boussinot, M. Apel, U. Hecht, Mushy zone solidification in a static thermal gradient: The effect of dendrite orientation. Materialia. 11, 100744 (2020)

A. Carré, B. Böttger, M. Apel, Implementation of an antitrapping current for a multicomponent multiphase-field ansatz. J. Cryst. Growth. 380, 5-13 (2013)

M. Chatelain, M. Albaric, D. Pelletier, V. Botton, Solute segregation in directoinal solidification : Scaling analysis of the solute boundary layer coupled with transient hydrodynamic simulations. J. Cryst. Growth. 430, 138-147 (2015)

D. C. Curtolo, G. S. Nayak, B. Friedrich, Definition of a first process window for purification of aluminum via cooled finger crystallization technique. Metals. 341(7) (2017). https://doi.org/10.3390/met7090341

J. Dantzig, M. Rappaz, Solidification, 1sted. (EPFL Press, 2009)

J. Eiken, Numerical solution of the phase-field equation with minimized discretization error. IOP Conf. Ser. Mater. Sci. Eng. 33, 012105 (2012). https://doi.org/10.1088/1757-899X/33/1/012105

J. Eiken, B. Böttger, I. Steinbach, Multiphase-field approach for multicomponent alloys with extrapolation scheme for numerical application. Phys. Rev. E. 73(6), 066122 (2006). https://doi.org/10.1103/PhysRevE.73.066122

S. Friedrich, D. C. Coladetti, B. Friedrich, Effect of process parameter variation on purity during rotary fractional crystallization of aluminium. Open J. Met. 25(7) (2017). https://doi.org/10.4236/ojmetal.2017.72003

G. Guillemot, C.h.-A. Gandin, Morphological stability of spherical particles - extension of the Mullins-Sekerka criteria to multi-component alloys under a non-stationary diffusive regime. Acta Mat. 205, 116539 (2021). https://doi.org/10. 1016/j.actamat.2020.116539

W. Kurz, D. J. Fisher, Fundamentals of Solidification, 4th ed. (Trans Tech, 1998), pp. 120-126 
A. Lahiri, A. Choudhury, Theoretical and numerical investigation of diffusive instabilities in multi-component alloys. J. Cryst. Growth. 459, 1-12 (2017). https://doi.org/10.1016/j.jcrysgro.2016.11.046

R. W. Lewis, K. Morgan, H. R. Thomas, K. N. Seetharamu, The Finite Element Method in Heat Transfer Analysis. (Wiley, 1996) MICRESS 6.4 (2018). http://www.micress.de. Accessed 2021

D. A. Porter, K. E. Easterling, Phase Transformations in Metals and Alloys 2nd Ed. (Taylor \& Francis, 1992), pp. 209-215

W. H. Sun, L. J. Zhang, M. Wei, Y. Du, B. Y. Huang, Effect of liquid diffusion coefficients on microstructure evolution during solidification of al356.1 alloy. Trans. Nonferrous Met. Soc. China. 23(12), 3722-3728 (2013). https://doi.org/10.1016/ S1003-6326(13)62922-2

Thermo-Calc database TCAL6 (2019). https://www.thermocalc.com/. Accessed 1 July 2021

L. O. Wilson, On interpreting a quantity in the Burton, Prim and Slichter equation as a diffusion boundary layer thickness. J. Cryst. Growth. 44, 247-250 (1978)

X. Zhang, S. Friedrich, B. Friedrich, Production of high purity metals: A review on zone refining process. J. Crystallization Process. Technol. 8 (2018). https://doi.org/10.4236/jcpt.2018.81003

\section{Publisher's Note}

Springer Nature remains neutral with regard to jurisdictional claims in published maps and institutional affiliations.

\section{Submit your manuscript to a SpringerOpen ${ }^{\circ}$ journal and benefit from:}

- Convenient online submission

- Rigorous peer review

- Open access: articles freely available online

- High visibility within the field

- Retaining the copyright to your article

Submit your next manuscript at $\gg$ springeropen.com 